\title{
Arsenic impurities in GaN
}

Cite as: Appl. Phys. Lett. 76, 1009 (2000); https://doi.org/10.1063/1.125922

Submitted: 15 November 1999 . Accepted: 20 December 1999 . Published Online: 14 February 2000

Chris G. Van de Walle, and J. Neugebauer

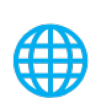

\section{ARTICLES YOU MAY BE INTERESTED IN}

Luminescence properties of defects in GaN

Journal of Applied Physics 97, 061301 (2005); https://doi.org/10.1063/1.1868059

First-principles calculations for defects and impurities: Applications to III-nitrides

Journal of Applied Physics 95, 3851 (2004); https://doi.org/10.1063/1.1682673

Gallium vacancies and the yellow luminescence in GaN

Applied Physics Letters 69, 503 (1996); https://doi.org/10.1063/1.117767

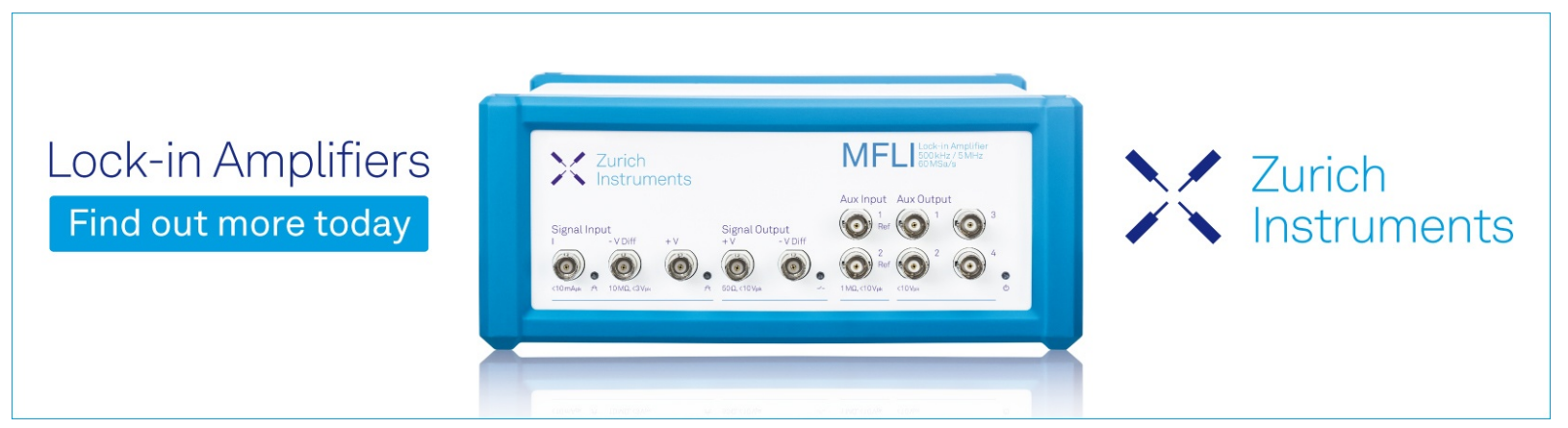




\title{
Arsenic impurities in GaN
}

\author{
Chris G. Van de Walle ${ }^{\mathrm{a})}$ \\ Fritz-Haber-Institut der Max-Planck-Gesellschaft, Faradayweg 4-6, D-14195 Berlin-Dahlem, Germany, and \\ Paul-Drude-Institut, Hausvogteiplatz 5-7, D-10117 Berlin, Germany \\ J. Neugebauer \\ Fritz-Haber-Institut der Max-Planck-Gesellschaft, Faradayweg 4-6, D-14195 Berlin-Dahlem, Germany
}

(Received 15 November 1999; accepted for publication 20 December 1999)

\begin{abstract}
We present a comprehensive first-principles investigation of arsenic incorporation in GaN. Incorporation of As on the $\mathrm{N}$ site, which has previously been implicitly assumed, is favorable only under $n$-type conditions in a Ga-rich environment. Less Ga-rich conditions, and particularly $p$-type doping, strongly favor incorporation of As on the Ga site, where it behaves as a deep double donor. Arsenic thus acts as a compensating center, forming a real threat to acceptor doping of $\mathrm{GaN}$ and making $p$-type doping of GaAsN alloys impossible. The calculated donor levels for $\mathrm{As}_{\mathrm{Ga}}$ are consistent with luminescence lines around $2.6-2.7 \mathrm{eV}$ in $\mathrm{GaN}$ intentionally doped with As. (C) 2000 American Institute of Physics. [S0003-6951(00)00508-8]
\end{abstract}

The interaction between arsenic and GaN has long been recognized to be important for materials properties of the nitride semiconductors. Significant attention has been devoted to the formation of GaAsN alloys. ${ }^{1-3}$ This ternary alloy system has been found to exhibit very limited solubility. Still, because of the large band-gap bowing ${ }^{3-5}$ even the addition of a few percent of As to GaN could result in a sizable modification of the band gap. Incorporation of As in $\mathrm{GaN}$ has also been investigated in the dilute limit. Theoretically, the behavior of the isolated As isoelectronic impurity and As-As pairs was investigated by Mattila and Zunger. ${ }^{6,7}$ Experimentally, incorporation of As during metalorganic chemical vapor deposition (MOCVD) and its effects on transport and optical properties were investigated by Guido et $a .^{8}$ and by Li et al. ${ }^{9}$ In addition to the intentional incorporation of As, unintentional exposure to As impurities has also been found to affect nitrides. This is particularly an issue in molecular-beam epitaxy (MBE), where previous growth of arsenides in the chamber results in a high As background. The presence of As has been found to completely modify the surface reconstructions observed for MBE-grown samples. ${ }^{10,11}$

It has generally been implicitly assumed that As would incorporate on the substitutional nitrogen site in bulk GaN. Indeed, being an element from column $\mathrm{V}$ of the periodic table, it seems natural that As would incorporate as an isoelectronic impurity on the nitrogen site. A large size mismatch exists, however, between nitrogen and arsenic: the covalent radius of $\mathrm{N}$ is $0.75 \AA$, while that of $\mathrm{As}$ is $1.20 \AA^{12}$ Based solely on size arguments, one might expect that As would more easily incorporate on the Ga site in GaN. However, on this site, an "electronic mismatch" obviously occurs, since we are placing a column- $\mathrm{V}$ element on a columnIII site. This is an "antisite"-type defect, and we therefore expect deep levels to occur in the band gap. The electronic behavior of this $\mathrm{As}_{\mathrm{Ga}}$ impurity can be conjectured from the

\footnotetext{
${ }^{a)}$ Permanent address: Xerox Palo Alto Research Center, 3333 Coyote Hill Road, Palo Alto, CA 94304; electronic mail: vandewalle@parc.xerox.com
}

fact that As occurs two columns to the right of $\mathrm{Ga}$ in the periodic table: it should therefore behave as a double donor.

These qualitative observations (ease of incorporation on the Ga site, and behavior as a double donor) will indeed turn out to be correct, but a quantitative determination is essential in order to assess the extent to which As will prefer the Ga site over the $\mathrm{N}$ site, if at all. Such a quantitative determination is now possible using state-of-the-art first-principles calculations. Using this approach, we find that while in $n$-type material (Fermi level $E_{F}$ above $2.3 \mathrm{eV}$ ), As incorporates on the $\mathrm{N}$ site, in semi-insulating or $p$-type material $\left(E_{F}\right.$ below $2.3 \mathrm{eV}$ ) As shows a strong preference for the Ga site. When incorporated on this site, As does not contribute to the formation of GaAsN alloys. Even worse, from an electronic point of view, is that the $\mathrm{As}_{\mathrm{Ga}}$ impurity behaves as a double donor. The unintentional presence of As during growth of acceptor-doped GaN will therefore result in significant compensation.

Our quantitative investigation of As incorporation in $\mathrm{GaN}$ is based on a formalism ${ }^{13,14}$ in which the equilibrium concentration of an impurity with formation energy $E^{f}$ is given by

$$
c=N_{\text {sites }} \exp \left(-E^{f} / k T\right) .
$$

The formation energy thus provides direct information about the tendency for an impurity to incorporate during growth. A general definition was given in Ref. 14; here, we focus on the specific case of As on a $\mathrm{Ga}$ site in $\mathrm{GaN}$, in charge state $q$ :

$$
\begin{aligned}
E^{f}\left(\operatorname{As}_{\mathrm{Ga}}^{q}\right)= & E^{\mathrm{tot}}\left(\mathrm{GaN}: \mathrm{As}_{\mathrm{Ga}}^{q}\right)-E^{\mathrm{tot}}(\mathrm{GaN}) \\
& -\mu_{\mathrm{As}}+\mu_{\mathrm{Ga}}+q E_{F} .
\end{aligned}
$$

$E^{\text {tot }}\left(\mathrm{GaN}: \mathrm{As}_{\mathrm{Ga}}^{q}\right)$ is the total energy (calculated from first principles as described below) of a system consisting of a part of a GaN crystal, with one of the Ga atoms replaced by As. To account for the removal of the Ga, we need to add the Ga chemical potential $\mu_{\mathrm{Ga}}$, which is the energy of the reservoir in which the Ga atom is placed. Similarly, the As atom is taken from a reservoir with energy $\mu_{\mathrm{As}}$. The Fermi energy 


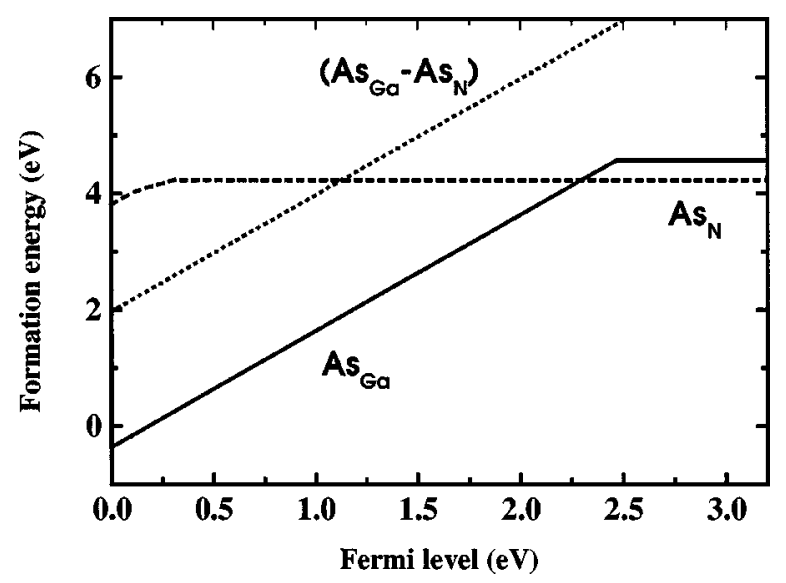

FIG. 1. Formation energies of As impurities in GaN as a function of Fermi level, obtained from first-principles calculations for the wurtzite phase, under Ga-rich conditions, and assuming equilibrium with GaAs. For each configuration, only the line segment corresponding to the charge state that gives rise to the lowest energy at a particular value of $E_{F}$ is shown; the slope of the line segment is determined by the charge state [see Eq. (2)]. The zero of Fermi energy is chosen at the top of the valence band, and the range of Fermi levels spans the theoretical band gap.

$E_{F}$ (set to zero at the valence-band maximum) enters because the center can occur in various charge states, and the electrons that are removed from the impurity need to be placed in their reservoir, with energy $E_{F}$.

The appearance of the chemical potentials in Eq. (2) corresponds to the experimental fact that growth can occur in Ga-rich vs N-rich conditions, and with different levels of As in the environment. In thermal equilibrium, the chemical potentials for $\mathrm{Ga}$ and $\mathrm{N}$ are related by $\mu_{\mathrm{Ga}}+\mu_{\mathrm{N}}=\mu_{\mathrm{GaN}}$. For displaying our results below, we choose Ga-rich growth conditions $\left(\mu_{\mathrm{Ga}}=\mu_{\mathrm{Ga}(\mathrm{bulk})}\right)$. This choice obviously disfavors incorporation of As on the Ga site. Even so, we will see that As still shows a strong tendency to do so, and this tendency can only be stronger if different growth conditions prevail. For As we choose the conditions corresponding to the solubility limit, imposed by the formation of GaAs, for which the expression $\mu_{\mathrm{Ga}}+\mu_{\mathrm{As}}=\mu_{\mathrm{GaAs}}$ holds. Our choice of the Garich growth conditions, combined with the calculated energy of bulk GaAs, then yields the value of $\mu_{\mathrm{As}}$.

The first-principles calculations from which we derive the energies needed in Eq. (2) are based on densityfunctional theory within the local density approximation (LDA) and the pseudopotential-plane-wave method. ${ }^{15}$ The pseudopotentials were created using the scheme of Troullier and Martins. ${ }^{16}$ The size of the supercells was 64 atoms for the zinc-blende (ZB) structure, and 96 atoms for the wurtzite (WZ) structure. Effects of the Ga3d states were included using the nonlinear core correction $(n l c c),{ }^{17}$ using an energy cutoff of $40 \mathrm{Ry}$. Explicit inclusion of the Ga $3 d$ electrons as valence electrons (with a 70 Ry cutoff) affected the formation energies by several $0.1 \mathrm{eV}$, but in a systematic fashion largely attributable to the difference in formation enthalpies of the bulk phases, and without affecting the qualitative conclusions of our study. The results reported below are for the $n l c c$.

Our main results are shown in Fig. 1, displaying the formation energies of the various species as a function of Fermi level. For Fermi level positions above $\sim 2.3 \mathrm{eV}$, the
TABLE I. Calculated formation energies $E^{f}$ and lattice relaxations $\Delta d$ around $\mathrm{As}_{\mathrm{Ga}}$ and $\mathrm{As}_{\mathrm{N}}$ impurities in zinc-blende and wurtzite $\mathrm{GaN}$, for various charge states. $E^{f}$ is given for a Fermi level at the top of the valence band. Off-center displacements of the impurity were allowed but found to be small $(<0.04 \AA)$. Changes in the bond length to nearest neighbors are expressed as a percentage of the equilibrium $\mathrm{Ga}-\mathrm{N}$ bond length; bonds parallel to the wurtzite $c$ axis are indicated by the symbol $\|$; the other bonds are indicated by $\perp$.

\begin{tabular}{crrrrrrr}
\hline \hline & \multicolumn{2}{c}{ Zinc blende } & & \multicolumn{4}{c}{ Wurtzite } \\
\cline { 2 - 3 } \cline { 5 - 7 } & \multicolumn{1}{c}{$\mathrm{E}^{f}(\mathrm{eV})$} & \multicolumn{1}{c}{$\Delta d$} & & $\mathrm{E}^{f}(\mathrm{eV})$ & $\Delta d_{\|}$ & $\Delta d_{\perp}$ \\
\hline $\mathrm{As}_{\mathrm{Ga}}^{0}$ & 4.72 & $+10.6 \%$ & & 4.57 & $+10.5 \%$ & $+10 \%$ \\
$\mathrm{As}_{\mathrm{Ga}}^{+}$ & 2.33 & $+4.3 \%$ & & 2.37 & $+4.1 \%$ & $+3.3 \%$ \\
$\mathrm{As}_{\mathrm{Ga}}^{++}$ & -0.63 & $-3.4 \%$ & & -0.37 & $-3.0 \%$ & $-3.6 \%$ \\
$\mathrm{As}_{\mathrm{N}}^{0}$ & 4.14 & $+15.5 \%$ & & 4.23 & $+16.0 \%$ & $+15.5 \%$ \\
$\mathrm{As}_{\mathrm{N}}^{+}$ & 3.67 & $+17.2 \%$ & & 3.92 & $+17.1 \%$ & $+17.3 \%$ \\
$\mathrm{As}_{\mathrm{N}}^{++}$ & 3.35 & $+18.6 \%$ & & 3.81 & $+18.0 \%$ & $+18.6 \%$ \\
\hline \hline
\end{tabular}

lowest-energy configuration corresponds to As on a nitrogen site, in a neutral charge state (as expected for an isoelectronic impurity). The fact that As is "too large" to be easily incorporated on this site is reflected in the large outward motion (see Table I) of the surrounding $\mathrm{N}$ atoms. The formation energy of $\mathrm{As}_{\mathrm{N}}$ is very high $(4.2 \mathrm{eV})$; in thermal equilibrium, only a very small concentration of As would be incorporated in this configuration. This is consistent with the observed low efficiency of As incorporation in (unintentionally $n$ type) MOCVD-grown $\mathrm{GaN}^{8,9}$ and with the known difficulties in growing GaAsN alloys. $\mathrm{As}_{\mathrm{N}}$ can change its charge state when the Fermi level is near the valence band; the Fermilevel positions at which the charge state changes correspond to the transition states: $\epsilon(2+/+)=0.11 \mathrm{eV}$ and $\epsilon(+/ 0)$ $=0.31 \mathrm{eV}$. These calculated deep donor levels are in good agreement with the results of Mattila and Zunger. ${ }^{6}$ The existence of $\mathrm{As}_{\mathrm{N}}$ in the + and $2+$ charge states does not affect our conclusions, as we will see below.

Figure 1 shows that the formation energy for $\mathrm{As}_{\mathrm{Ga}}$ in the neutral charge state is $0.3 \mathrm{eV}$ higher than for $\mathrm{As}_{\mathrm{N}}{ }^{0}$. However, the values in Fig. 1 were derived for Ga-rich conditions. Less Ga-rich growth conditions lower the formation energy of $\mathrm{As}_{\mathrm{Ga}}$, and raise that of $\mathrm{As}_{\mathrm{N}}$. More importantly, we note that the behavior of $\mathrm{As}_{\mathrm{Ga}}$ as a deep donor significantly lowers its formation energy in the + and $2+$ charge states. For Fermi levels below about $2.3 \mathrm{eV}, \mathrm{As}_{\mathrm{Ga}}^{2+}$ is by far the most favorable configuration, with a formation energy that becomes very low under $p$-type conditions. This result shows that when As is present while growing acceptor-doped $\mathrm{GaN}$, it will readily incorporate on a Ga site and behave as a deep donor, compensating the acceptors. It also shows that $p$-type doping of GaAsN alloys will be impossible, since the As that is present to form the alloy forms an exceedingly effective compensating center for acceptors.

For $\mathrm{As}_{\mathrm{Ga}}$, the transition levels occur at $\epsilon(2+/+)$ $=2.7 \mathrm{eV}$, and $\epsilon(+/ 0)=2.2 \mathrm{eV}$. Note that the ordering of these levels is unusual: adding electrons to a level usually causes the level to go up in energy, because of Coulomb repulsion $(U)$. For $\mathrm{As}_{\mathrm{Ga}}$, however, the $\epsilon(+/ 0)$ level occurs below the $\epsilon(2+/+)$ level, which is characteristic of a socalled "negative- $U$ " center. Negative- $U$ centers typically involve large lattice relaxations; Table I indeed shows that the relaxations of the neighboring $\mathrm{N}$ atoms are large, and 
very different in different charge states (changing from $+11 \%$ to $-3 \%)$. The particular ordering of the $\epsilon(2+/+)$ and $\epsilon(+/ 0)$ transition levels implies that in thermal equilibrium the + charge state will never be stable (as can be seen from Fig. 1).

The error bar on the calculated transition levels is quite large, due to the well-documented LDA band-gap error. However, the qualitative result that $\mathrm{As}_{\mathrm{Ga}}$ acts as a deep donor, with negative- $U$ character, is firm. The defect wave function is mainly composed of $p$ states on the neighboring $\mathrm{N}$ atoms, and therefore exhibits valence-band character. The position of the defect level is thus expected to be fixed with respect to the valence band, even when corrections to the LDA band gap are applied. This observation is supported by a comparison between calculations using the nonlinear core correction and explicit inclusion of $3 d$ states: the defect levels occur at very similar energies above the valence-band maximum, in spite of the very different theoretical band gaps (3.03 eV with nlcc vs $1.61 \mathrm{eV}$ with $3 d$ for zinc-blende $\mathrm{GaN})$.

Several groups have reported luminescence lines around 2.6-2.7 eV in intentionally As-doped samples. Pankove and Hutchby ${ }^{18}$ reported a peak at $2.58 \mathrm{eV}$ for samples that were As-implanted and annealed. Li et al. ${ }^{9}$ observed a roomtemperature luminescence band around $2.6 \mathrm{eV}$, both in uniformly doped GaN:As and in GaN/GaAs superlattices. Guido et al., ${ }^{8}$ finally, observed a luminescence signal at about $2.73 \mathrm{eV}$ (at $77 \mathrm{~K}$ ); they noted the possibility of As incorporation on the Ga site, but provided no direct experimental evidence for this lattice location. The luminescence lines observed in these experiments cannot be explained by the donor levels of $\mathrm{As}_{\mathrm{N}}$, which occur at 0.11 and $0.31 \mathrm{eV}$ above the valence band. Instead, we suggest that these lines are due to $\mathrm{As}_{\mathrm{Ga}}$. The scatter in the experimental results and the large width of the observed lines are likely due to proximity of the impurity to other impurities or defects, as well as to the involvement of phonons due to the large lattice relaxations between different charge states.

For completeness, we have also carried out calculations for other configurations involving As. Arsenic placed on various interstitial sites, even allowing low-symmetry configurations, is found to be much higher in energy (by at least $1 \mathrm{eV}$, for all charge states) than the highest-energy configurations depicted in Fig. 1. Finally, we have considered $\mathrm{As}_{\mathrm{Ga}}-\mathrm{As}_{\mathrm{N}}$ complexes. We find that complexes in which two As atoms are nearest neighbors can be formed with a positive binding energy. In the $2+$ charge state, this binding energy is $\sim 1.9 \mathrm{eV}$. This binding energies is one order of magnitude larger than that reported for $\mathrm{As}_{\mathrm{N}}-\mathrm{As}_{\mathrm{N}}$ pairs in Ref. 6; however, we note that even a binding energy of $2 \mathrm{eV}$ is not sufficient to compensate for the very high formation energy of the $\mathrm{As}_{\mathrm{N}}$ species. Formation of the isolated $\mathrm{As}_{\mathrm{Ga}}$ center will thus still be favored over formation of an $\left(\mathrm{As}_{\mathrm{Ga}}-\mathrm{As}_{\mathrm{N}}\right)$ complex.

In summary, we have reported comprehensive calculations for the behavior of As impurities in $\mathrm{GaN}$, focusing particular attention on the previously unexplored incorporation of As on a Ga site. We find this configuration behaves as a deep donor, with a very low formation energy under $p$-type conditions. It will thus form a significant source of compensation in acceptor-doped GaN, and render $p$-type doping of GaAsN impossible. Even under $n$-type conditions, the formation energy of $\mathrm{As}_{\mathrm{Ga}}$ is comparable to $\mathrm{As}_{\mathrm{N}}$; incorporation of As on the Ga site thus competes with incorporation on the $\mathrm{N}$ site, which would be required for growth of GaAsN alloys. Our conclusions indicate that $n$-type conditions (i.e., doping with donors) serves to stabilize GaAsN alloys. Our results highlight the difficulties to be encountered during intentional incorporation of As to form GaAsN alloys, as well as the danger of having As present during growth of $p$-type GaN.

One of the authors' stay (C.VdW.) in Berlin was enabled by a U.S. Senior Scientist Award from the Alexander von Humboldt Foundation.

\footnotetext{
${ }^{1}$ M. Weyers, M. Sato, and H. Ando, Jpn. J. Appl. Phys., Part 2 31, L853 (1992).

${ }^{2}$ J. Neugebauer and C. G. Van de Walle, Phys. Rev. B 51, 10568 (1995).

${ }^{3}$ S.-H. Wei and A. Zunger, Phys. Rev. Lett. 76, 664 (1996).

${ }^{4}$ J. Neugebauer and C. G. Van de Walle, Mater. Res. Soc. Symp. Proc. 379, 3 (1995)

${ }^{5}$ A. Rubio and M. L. Cohen, Phys. Rev. B 51, 4343 (1995).

${ }^{6}$ T. Mattila and A. Zunger, Phys. Rev. B 58, 1367 (1998).

${ }^{7}$ T. Mattila and A. Zunger, Phys. Rev. B 59, 9943 (1999).

${ }^{8}$ L. J. Guido, P. Mitev, M. Gherasimova, and B. Gaffey, Appl. Phys. Lett. 72, 2005 (1998)

${ }^{9}$ X. Li, S. Kim, E. E. Reuter, S. G. Bishop, and J. J. Coleman, Appl. Phys. Lett. 72, 1990 (1998).

${ }^{10}$ G. Feuillet, H. Hamaguchi, K. Ohta, P. Hacke, H. Okumura, and S. Yoshida, Appl. Phys. Lett. 70, 1025 (1997).

${ }^{11}$ J. Neugebauer, T. Zywietz, M. Scheffler, J. E. Northrup, and C. G. Van de Walle, Phys. Rev. Lett. 80, 3097 (1998).

${ }^{12}$ Periodic Table of the Elements, Sargent-Welch Scientific Company.

${ }^{13}$ J. Neugebauer and C. G. Van de Walle, Phys. Rev. B 50, 8067 (1994).

${ }^{14}$ J. Neugebauer and C. G. Van de Walle, in Festkörperprobleme/Advances in Solid State Physics, edited by R. Helbig (Vieweg, Braunschweig/ Wiesbaden, 1996), Vol. 35, p. 25.

${ }^{15}$ M. Bockstedte, A. Kley, J. Neugebauer, and M. Scheffler, Comput. Phys. Commun. 107, 187 (1997).

${ }^{16}$ N. Troullier and J. L. Martins, Phys. Rev. B 43, 1993 (1991).

${ }^{17}$ S. G. Louie, S. Froyen, and M. L. Cohen, Phys. Rev. B 26, 1738 (1982).

${ }^{18}$ J. I. Pankove and J. A. Hutchby, J. Appl. Phys. 47, 5387 (1976).
} 